\title{
Subacute Oral Toxicity and Bacterial Mutagenicity Study of a Mixture of Korean Red Ginseng (Panax ginseng C.A. Meyer) and Salvia plebeia R. Br. Extracts
}

\author{
Hwi Won Seo ${ }^{\dagger}$, Jae Hyun Suh ${ }^{\dagger}$, Jong-Soo Kyung, Kyoung Hwa Jang and Seung-Ho So \\ Laboratory of Fundamental Research, Korea Ginseng Corporation, Daejeon, Korea
}

\begin{abstract}
As various populations are rapidly becoming an aging society worldwide and interest in health issues has increased, demand for functional foods including herbal products has increased markedly to maintain a healthy state which has led to safety issues about their intake as an inevitable result. The objective of this study was to identify the safety profile of a Korean red ginseng and Salvia plebeia R. Br. extract mixture (KGC-03-PS) which is a valuable ingredient that can be used as a functional food. In the present study, the subacute oral toxicity and bacterial reverse mutagenicity of KGC-03-PS were evaluated. Sprague Dawley rats were administered KGC-03PS orally for 28 days by gavage. Daily KGC-03-PS dose concentrations were $0,500,1,000$, or 2,000 mg/kg body weight (bw) per day. Bacterial reverse mutation test with KGC-03-PS dose levels ranging from 312.5 to $5,000 \mu \mathrm{g} /$ plate was carried out by OECD test guideline No. 471. Five bacterial strains (Salmonella typhimurium TA98, TA100, TA1535, TA1537, and Escherichia coli WP2) were tested in the presence or absence of metabolic activation by plate incorporation method. There were no toxicological effects related with test substance in the clinical evaluation of subacute oral toxicity test including clinical signs, body weight, and food consumption. Moreover, no toxicological changes related to KGC-03-PS were observed in the hematological and serum biochemical characteristics as well as in the pathological examinations, which included organ weight measurements and in the gross- or histopathological findings. KGC-03-PS did not induce an increase in the number of revertant colonies in all bacterial strains of the bacterial reverse mutation test. The no-observed-adverse-effect level of KGC-03-PS is greater than $2,000 \mathrm{mg} / \mathrm{kg}$ bw/day, and KGC-03-PS did not induce genotoxicity related to bacterial reverse mutations under the conditions used in this study.
\end{abstract}

Key words: Korean red ginseng and Salvia plebeia R. Br. extract mixture (KGC-03-PS), Subacute oral toxicity, Bacterial reverse mutagenicity, No-observed-adverse-effect level

\section{INTRODUCTION}

As the consumption of natural functional foods has markedly increased, cases of adverse reactions related to intake safety have also increased (1). Moreover, it is more diffi-

\footnotetext{
Correspondence to: Seung-Ho So, Laboratory of Fundamental Research, Korea Ginseng Corporation, 30, Gajeong-ro, Shinseongdong, Yuseong-gu, Daejeon 34128, Korea

E-mail: biopro@kgc.co.kr

${ }^{\dagger}$ The first two authors contributed equally to this work.

This is an Open-Access article distributed under the terms of the Creative Commons Attribution Non-Commercial License (http:// creativecommons.org/licenses/by-nc/3.0) which permits unrestricted non-commercial use, distribution, and reproduction in any medium, provided the original work is properly cited.
}

cult to determine the safety of natural ingredients than that of drugs, which have a single active pharmaceutical ingredient because natural ingredients have various constituents, and most functional foods or supplements are a mixture of various ingredients (2).

Therefore, scientific evidence on the safety of ingredients in natural foods or supplementary diets is required, and experimental studies in vivo and in vitro have been done according to guidelines established by international organizations such as the Organization for Economic Cooperation and Development (OECD) and the International Council for harmonisation (ICH). In particular, general toxicity and genotoxicity studies are representative of what related authorities require for determining the safety of ingredients.

Korean red ginseng (Panax ginseng C.A. Meyer), pro- 
duced by steaming Panax ginseng in water vapor, has been traditionally used as medicine and supplementary diet in Asian countries including Korea (3). It contains ginsenosides well known as a main biological component in red ginseng as well as arginine-fructose-glucose, acidic polysaccharides, and poly acetylenes whose biological activities have been demonstrated in previous studies (4-8). It has also been reported that Korean red ginseng has various pharmaceutical potencies including neurological improvement (9), blood pressure regulation (10), anti-inflammatory (11), anti-cancer (12), and liver protection (13).

Salvia plebeia $\mathrm{R}$. Br. belongs to the family Lamiaceae, and is an annual herb widely distributed in many counties including Korea, China, Japan, India, Iran, and Australia. It has been used as a traditional medicine to treat various inflammatory diseases including hepatitis, cough, tumors, diarrhea (14), and atopic dermatitis (15), and it was also shown to have a strong anti-oxidative activity $(16,17)$, anti-obesity effects in mice fed high-fat diets (18), and a respiratory protective effect in ambient particulate matterinduced airway inflammation (19).

Korean red ginseng is often used in mixtures with other herbs which could be used to enhance the effects of red ginseng (20). Because the Korean red ginseng and Salvia Salvia plebeia $\mathrm{R}$. Br. extract has strong anti-inflammation and anti-oxidation effects, the mixture could also be developed as a functional food or supplementary diet ingredient with the expectation of a synergistic effect between the two herbs.

Despite the usefulness of the Korean red ginseng and Salvia plebeia $\mathrm{R}$. Br. extract mixture, there has been little published scientific evidence on it, and what has been published about the toxicity of Korean red ginseng $(21,22)$ is not sufficient to ensure the intake safety of each ingredient alone or as a mixture.

Therefore, we did repeated-dose oral administration and bacterial reverse mutagenicity studies to identify the potential toxicity of the Korean red ginseng and Salvia plebeia R. Br. extract mixture.

\section{MATERIALS AND METHODS}

Test substance and anaylsis. The extract powder of two herbs was mixed in a ratio of Korean red ginseng: Salvia plebeia $\mathrm{R}$. Br., 1:3 which was determined as optimal composition for anti-oxidant and anti-inflammatory effects in vitro and in vivo preliminary tests. Korean red ginseng extract powder was provided from Korea Ginseng Corporation and dried Salvia plebeia $\mathrm{R}$. Br. was provided from Buan Dongjin Farm (Buan, Korea). Salvia plebeia $\mathrm{R}$. Br. were extracted two times with an fifteenfold volume of $30 \%$ alcohol for $4 \mathrm{hr}$ at $80^{\circ} \mathrm{C}$. After the extract was filtered through $1 \mu \mathrm{m}$ pore size, the resultant was concentrated on evaporator and prepared into powder
Table 1. Contents of test article KGC-03-PS

\begin{tabular}{cc}
\hline \hline Component & Content $(\mathrm{mg} / \mathrm{g})$ \\
\hline $\mathrm{Rg} 1$ & 0.45 \\
$\mathrm{Rb} 1$ & 1.96 \\
$\mathrm{Rg} 3(\mathrm{~S})$ & 0.65 \\
Nepetin & 3.06 \\
\hline
\end{tabular}

by spray-drying. Quantities of analytical markers in Korean red ginseng and Salvia plebeia R. Br. extract mixture (KGC03-PS) was analyzed by ultra performance liquid chromatography (UPLC) using Waters BEH C18 column $(1.7 \mu \mathrm{m}$, $2.1 \times 50 \mathrm{~mm}$ ). The composition of KGC-03-PS was showed in Table 1. The distilled water was used for administration vehicle.

Subacute oral toxicity study. Subacute oral toxicity test were based on the Organisation for Economic Co-operation and Development (OECD) - Guideline 407 (23).

- Test animals and environmental conditions: Fiveweeks-old male and female, specific pathogen-free (SPF) Sprague-Dawley (SD) rats were purchased from Koatech Inc. (Pyeongtaek, Korea) and acclimated for 7 days before first administration. The rats were housed in polycarbonate plastic cages ( 1 rat per cage) with aspen porous GLP bedding (Samtako, Osan, Korea) in a room with controlled temperature $\left(20-23^{\circ} \mathrm{C}\right)$ and humidity (40-55\%), and a $12 \mathrm{hr}$ light/dark cycle. The rats were fed standard rodent chow (Purina, MN, USA) and filtered water ad libitum. This experiment was approved by Institutional Animal Care and Use Committee of KT\&G.

- Experimental group: At six weeks old, the rats were divided into four groups (6 male and 6 female rats in each group): vehicle control (distilled water), low-dose group (500 mg/kg/day), middle-dose group $(1,000 \mathrm{mg} / \mathrm{kg} /$ day), and high-dose group $(2,000 \mathrm{mg} / \mathrm{kg} / \mathrm{day})$. The rats were exposed to KGC-03-PS following the toxicity test guideline from the Korea Food and Drug Administration (KFDA) for Nonclinical Laboratory Studies. The dosing volumes were $10 \mathrm{~mL} / \mathrm{kg}$ bw and the route of administration was oral. The maximum dose was determined according to the recommendation by the OECD - Guideline 423 (24).

- Body weight changes: Clinical signs of animals were observed each administration day during the $30 \mathrm{~min}$ after dosing. Body weight of animals was measured weekly from the initiation day of administration to the day of necropsy.

- Food consumption: Food consumption was measured at the initiation day of administration, thereafter weekly, and the day of last administration, and then calculated the daily average food intake.

- Biochemistry and hematology: Before necropsy, all rats were fasted for overnight and killed by exsanguina- 
tions under isoflurane anesthesia after recording the body weights. Blood samples were drawn from the inferior vena cava, collected in EDTA-vacutainers, and analyzed for the white blood cell (WBC), red blood cell (RBC), hemoglobin (HB), hematocrit (HCT), mean corpuscular volume (MCV), mean corpuscular hemoglobin (MCH), mean corpuscular hemoglobin concentration (MCHC), and platelet counts (PLT) using a blood cell counter (MS9-5, Melet Schloesing laboratories, Osny, France).

For clinical biochemistry analyses, serum was obtained from collected blood in a EDTA-free vacutainer by centrifugation at 3,000 rpm for $10 \mathrm{~min}$. Biochemical automatic analyzer (7100, HITACHI, Tokyo, Japan) were used for analyzing alanine aminotransferase (ALT), aspartate aminotransferase (AST), alkaline phosphatase (ALP), blood urea nitrogen $(\mathrm{BUN})$, creatinine (CRE), total protein (TP), albumin (ALB), A/G ratio, total cholesterol (T-CHO), triglyceride (TG), and glucose (GLU).

- Organ weights: The organ including the brain, heart, lung, liver, spleen, kidneys, adrenal glands, testis, and uterus were dissected and the absolute weights were measured immediately. Relative organ weights were calculated as
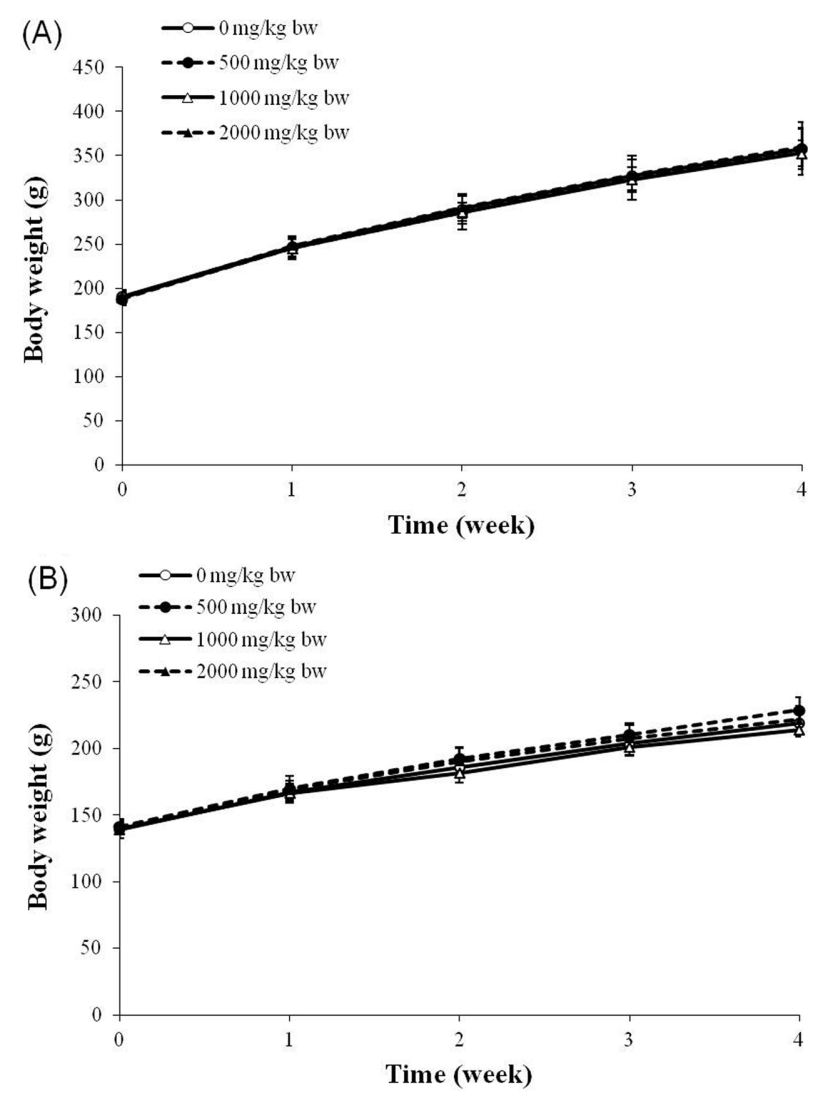

Fig. 1. Body weight changes of male (A) and female (B) rats in the 28 days oral administration toxicity test. The rats exposed to KGC-03-PS showed no significant difference compared to control. the ratio between the absolute organ weight and the body weight before necropy.

- Histopathology: The macroscopic organ observation was checked in all animals. The heart, lung, liver, spleen and kidneys were fixed in $10 \%$ neutral buffered formalin. Each fixed organ was trimmed as described in previous guideline (25). After paraffin infiltration by tissue processor, the organs were embedded in paraffin and cut into $4 \mu \mathrm{m}$ section. The sections were stained with hematoxylin and eosin, and examined under light microscopy.

Bacterial reverse mutation test. The bacterial reverse mutation test (also called Ames test) was carried out according to the OECD - Guideline 471 for the testing of chemicals "Bacterial reverse mutation test" (26).

- Tester strains: In the test, five strains such as TA98, TA100, TA1535, TA1537 of Salmonella typhimurium, and WP2uvrA of Escherichia coli were used. These strains are highly sensitive to mutagens, commonly used in mutagenicity studies and recommended in the regulatory guidelines (27). Salmonella typhimurium TA100, TA1535 and E. coli WP uvrA were used to detect the base-pair substi-
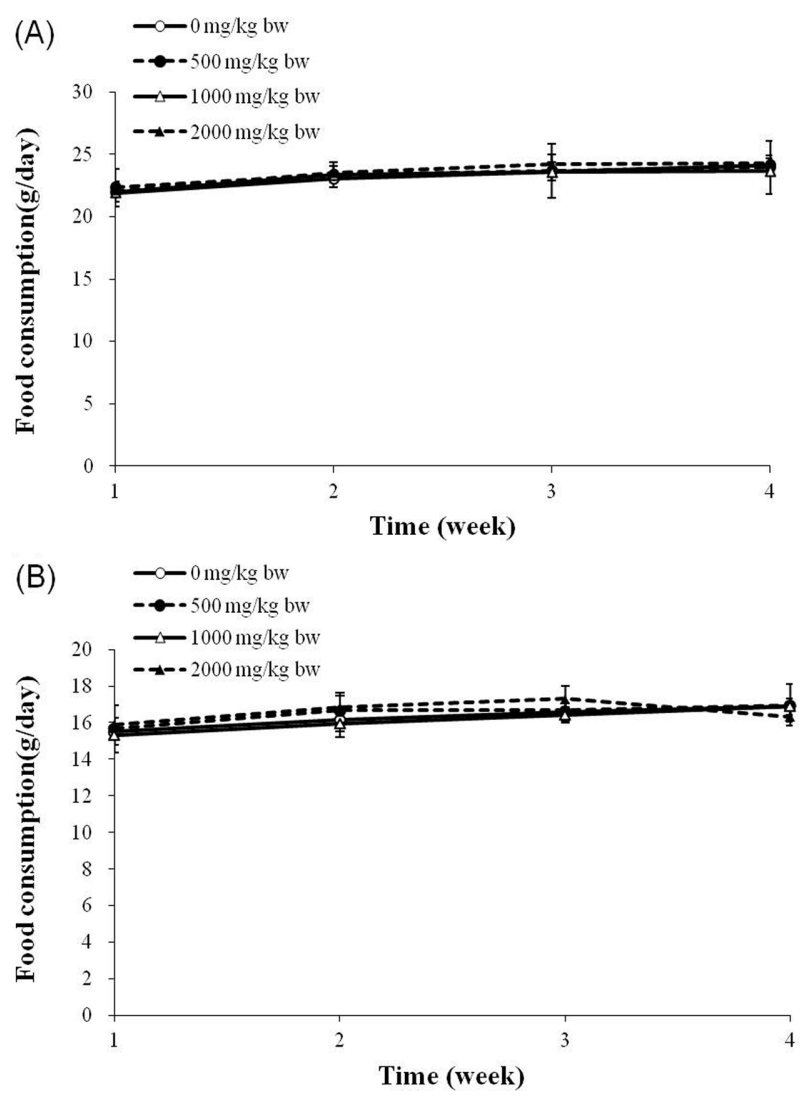

Fig. 2. Food consumption of male (A) and female (B) rats in the 28 days oral administration toxicity test. The rats exposed to KGC-03-PS showed no significant difference compared to control. 
tution type mutation, whereas TA98 and TA1537 were used to detect the frame-shift type mutation (28-30). The Salmonella strains were purchased from Molecular Toxicology Inc. and $E$. coli WP2 uvrA strain was obtained from Preclinical Research Center (ChemOn, Inc., Gyeonggi, Korea).

- Controls: With metabolic activation system, Benzo $[\alpha]$ pyrene (Sigma-Aldrich, MO, USA) for TA98, 2Aminoanthracene (Sigma-Aldrich) for TA100, TA1535, TA1537 and E. coli. Without metabolic activation system, Sodium azide (Sigma-Aldrich) for TA100 and TA1535, 4Nitroquinoline N-oxide (Sigma-Aldrich) for TA98 and $E$. coli, 9-Aminoacridine (Sigma-Aldrich) for TA1537 were used.

- Test substance treatment: As Salvia plebeian leaf extracts in KGC-03-PS was extracted with 30\% ethanol, test article was diluted in $30 \%$ ethanol. $30 \%$ ethanol was used as negative control.

- Treatment method: According to the results of a dose finding test, $5,000 \mu \mathrm{g} /$ plate was selected as the highest concentration for all test strains both the absence and presence of S9 mixture.
For the test, two-fold serial dilutions were performed to yield five concentration $(312.5,625,1,250,2,500$, and $5,000 \mu \mathrm{g} /$ plate).

The test was performed based on the plate incorporation method with or out metabolic activation system S9 mixture $(31,32)$. Each sample was assayed in triplicate. Each culture plate was placed in incubator for $48 \mathrm{hr}$ at $37^{\circ} \mathrm{C}$. After the incubation, the number of revertant colonies was counted by visual counting.

- Evaluation and interpretation of results: The test substance was considered as positive in the bacterial reverse mutation assay when there was an increase ( $\geq$ twofold) of spontaneous revertant colonies compared with those in the negative control or a concentration-related increase over the range tested and/or a reproducible increase at one or more concentrations in the number of revertant colonies per plate in at least one strain with or without metabolic activation.

Statistical analysis. The data for the body weights, food consumption, and organ weights and the blood biochemistry and hematology indexes were analyzed using an

Table 2. Blood biochemistry data for rats in the 28 days oral administration of KGC-03-PS

\begin{tabular}{|c|c|c|c|c|}
\hline & \multicolumn{4}{|c|}{ Dose $(\mathrm{mg} / \mathrm{kg})$} \\
\hline & $0(n=6)$ & $500(\mathrm{n}=6)$ & $1000(\mathrm{n}=6)$ & $2000(n=6)$ \\
\hline \multicolumn{5}{|l|}{ Male } \\
\hline ALB & $2.48 \pm 0.05$ & $2.51 \pm 0.04$ & $2.55 \pm 0.07$ & $2.50 \pm 0.10$ \\
\hline ALP & $395.0 \pm 91.2$ & $432.7 \pm 31.3$ & $398.7 \pm 48.2$ & $371.7 \pm 37.9$ \\
\hline T-CHO & $80.5 \pm 10.1$ & $76.0 \pm 3.9$ & $74.7 \pm 13.4$ & $84.3 \pm 5.6$ \\
\hline CRE & $0.48 \pm 0.04$ & $0.45 \pm 0.05$ & $0.47 \pm 0.05$ & $0.42 \pm 0.04$ \\
\hline GLU & $110.3 \pm 13.3$ & $112.8 \pm 10.2$ & $102.0 \pm 20.4$ & $107.5 \pm 24.4$ \\
\hline AST & $120.5 \pm 14.7$ & $120.3 \pm 17.7$ & $121.5 \pm 14.3$ & $112.8 \pm 13.5$ \\
\hline ALT & $43.8 \pm 10.2$ & $43.0 \pm 6.1$ & $39.5 \pm 3.8$ & $38.3 \pm 5.1$ \\
\hline $\mathrm{TP}$ & $5.55 \pm 0.10$ & $5.53 \pm 0.15$ & $5.62 \pm 0.13$ & $5.60 \pm 0.14$ \\
\hline BUN & $19.1 \pm 1.5$ & $18.6 \pm 2.2$ & $19.4 \pm 2.7$ & $17.6 \pm 1.0$ \\
\hline $\mathrm{A} / \mathrm{G}$ ratio & $0.81 \pm 0.02$ & $0.83 \pm 0.02$ & $0.83 \pm 0.02$ & $0.81 \pm 0.04$ \\
\hline TG & $38.8 \pm 7.0$ & $46.7 \pm 8.3$ & $39.8 \pm 10.5$ & $43.7 \pm 9.2$ \\
\hline \multicolumn{5}{|l|}{ Female } \\
\hline ALB & $2.62 \pm 0.09$ & $2.61 \pm 0.04$ & $2.65 \pm 0.11$ & $2.55 \pm 0.11$ \\
\hline ALP & $269.7 \pm 63.0$ & $306.5 \pm 61.2$ & $299.0 \pm 61.9$ & $243.0 \pm 24.2$ \\
\hline $\mathrm{T}-\mathrm{CHO}$ & $83.5 \pm 10.4$ & $91.5 \pm 11.8$ & $88.7 \pm 10.8$ & $79.7 \pm 13.0$ \\
\hline CRE & $0.47 \pm 0.05$ & $0.50 \pm 0.00$ & $0.50 \pm 0.06$ & $0.48 \pm 0.04$ \\
\hline GLU & $96.5 \pm 7.4$ & $101.7 \pm 8.3$ & $110.2 \pm 10.9$ & $113.2 \pm 13.5^{*}$ \\
\hline AST & $109.7 \pm 14.4$ & $109.5 \pm 12.0$ & $109.7 \pm 13.2$ & $97.7 \pm 13.2$ \\
\hline ALT & $30.7 \pm 1.8$ & $31.2 \pm 3.8$ & $30.3 \pm 3.8$ & $29.5 \pm 2.3$ \\
\hline $\mathrm{TP}$ & $5.57 \pm 0.15$ & $5.55 \pm 0.14$ & $5.58 \pm 0.17$ & $5.43 \pm 0.20$ \\
\hline BUN & $18.3 \pm 1.2$ & $18.2 \pm 2.9$ & $20.4 \pm 2.2$ & $18.9 \pm 2.5$ \\
\hline $\mathrm{A} / \mathrm{G}$ ratio & $0.89 \pm 0.02$ & $0.89 \pm 0.02$ & $0.90 \pm 0.04$ & $0.89 \pm 0.03$ \\
\hline TG & $23.7 \pm 6.8$ & $25.5 \pm 11.9$ & $22.0 \pm 7.1$ & $21.0 \pm 4.7$ \\
\hline
\end{tabular}

ALB (g/dL), Albumin; ALP (IU/L), Alkaline phosphatase; CHO (mg/dL), Total cholesterol; CRE (mg/dL), Creatinine; GLU (mg/dL), Glucose; AST (IU/L), Aspartate aminotransferase; ALT (IU/L), Alanine aminotransferase; TP (g/dL), Total protein; BUN (mg/dL), Blood urea nitrogen; A/G ratio; TG (mg/dL), Triglyceride.

Data were expressed as mean \pm SD.

"Significant difference vs. control $(p<0.05)$. 
one-way analysis of variance (ANOVA) if the variances of the groups were assumed to be equal. When ANOVA showed a statistical significance, post-hoc Dunnett's multiple range test was used to identify the differences between the control and the experimental groups. If the variances of the groups were not assumed to be equal, Kruskal-Wallis test was used to determine the significance of the group differences. The IBM SPSS statistics version 20 software

Table 3. Hematological data for rat in the 28 days oral administration of KGC-03-PS

\begin{tabular}{|c|c|c|c|c|}
\hline & \multicolumn{4}{|c|}{ Dose (mg/kg) } \\
\hline & $0(n=6)$ & $500(\mathrm{n}=6)$ & $1000(\mathrm{n}=6)$ & $2000(n=6)$ \\
\hline \multicolumn{5}{|l|}{ Male } \\
\hline WBC & $8.33 \pm 1.40$ & $8.44 \pm 1.29$ & $8.71 \pm 1.81$ & $9.96 \pm 1.75$ \\
\hline $\mathrm{RBC}$ & $7.96 \pm 0.36$ & $8.66 \pm 0.48$ & $8.92 \pm 0.39^{*}$ & $8.86 \pm 0.73$ \\
\hline $\mathrm{Hb}$ & $17.62 \pm 0.57$ & $18.67 \pm 1.46$ & $19.48 \pm 1.60$ & $19.62 \pm 1.46$ \\
\hline HCT & $43.0 \pm 1.2$ & $46.2 \pm 3.4$ & $47.7 \pm 3.8$ & $47.8 \pm 2.8$ \\
\hline $\mathrm{MCV}$ & $54.2 \pm 1.1$ & $52.8 \pm 0.5$ & $52.9 \pm 1.6$ & $53.0 \pm 0.6$ \\
\hline $\mathrm{MCH}$ & $22.2 \pm 1.1$ & $21.4 \pm 0.7$ & $21.4 \pm 0.3$ & $21.9 \pm 0.8$ \\
\hline $\mathrm{MCHC}$ & $40.1 \pm 1.7$ & $40.5 \pm 1.0$ & $40.6 \pm 1.1$ & $41.2 \pm 1.2$ \\
\hline PLT & $761 \pm 53$ & $658 \pm 96$ & $653 \pm 81$ & $708 \pm 76$ \\
\hline \multicolumn{5}{|l|}{ Female } \\
\hline WBC & $6.08 \pm 0.95$ & $7.45 \pm 1.40$ & $7.48 \pm 1.96$ & $6.90 \pm 0.96$ \\
\hline $\mathrm{RBC}$ & $7.47 \pm 0.37$ & $7.43 \pm 0.28$ & $8.12 \pm 0.45$ & $8.24 \pm 0.62^{*}$ \\
\hline $\mathrm{Hb}$ & $15.9 \pm 0.7$ & $16.2 \pm 0.5$ & $16.7 \pm 0.8$ & $17.6 \pm 1.1^{*}$ \\
\hline HCT & $42.6 \pm 1.5$ & $41.9 \pm 2.6$ & $46.7 \pm 1.9$ & $47.8 \pm 3.5^{*}$ \\
\hline $\mathrm{MCV}$ & $57.2 \pm 2.3$ & $56.4 \pm 1.9$ & $57.5 \pm 1.6$ & $57.0 \pm 1.0$ \\
\hline $\mathrm{MCH}$ & $21.3 \pm 0.9$ & $21.9 \pm 0.5$ & $20.6 \pm 0.6$ & $21.4 \pm 0.6$ \\
\hline $\mathrm{MCHC}$ & $37.2 \pm 1.0$ & $38.9 \pm 1.5$ & $35.8 \pm 1.5$ & $37.6 \pm 0.7$ \\
\hline PLT & $915 \pm 48$ & $905 \pm 35$ & $898 \pm 95$ & $774 \pm 109^{*}$ \\
\hline
\end{tabular}

WBC $\left(10^{3} / \mu \mathrm{L}\right)$, White blood cells; RBC (10\% $\left./ \mu \mathrm{L}\right)$, Red blood cells; Hb (g/dL), Hemoglobin; HCT (\%), Hematocrits; MCV (fL), Mean corpuscular volume; $\mathrm{MCH}$ (pg), Mean corpuscular hemoglobin; $\mathrm{MCHC}(\mathrm{g} / \mathrm{dL})$, Mean corpuscular hemoglobin concentration; $\mathrm{PLT}\left(10^{3} / \mu \mathrm{L}\right), \mathrm{Platelet}$. Data were expressed as mean \pm SD.

"Significant difference vs. control $(p<0.05)$.

Table 4. Absolute organ weight for rats in the 28 days oral administration of KGC-03-PS

\begin{tabular}{|c|c|c|c|c|}
\hline \multirow{2}{*}{$\begin{array}{l}\text { Absolute weight } \\
\text { (g) }\end{array}$} & \multicolumn{4}{|c|}{ Dose $(\mathrm{mg} / \mathrm{kg})$} \\
\hline & $0(n=6)$ & $500(n=6)$ & $1000(n=6)$ & $2000(n=6)$ \\
\hline \multicolumn{5}{|l|}{ Male } \\
\hline Brain & $1.91 \pm 0.18$ & $1.87 \pm 0.12$ & $1.89 \pm 0.10$ & $1.96 \pm 0.04$ \\
\hline Heart & $1.13 \pm 0.11$ & $1.14 \pm 0.11$ & $1.19 \pm 0.06$ & $1.16 \pm 0.08$ \\
\hline Lung & $1.47 \pm 0.09$ & $1.50 \pm 0.11$ & $1.48 \pm 0.12$ & $1.45 \pm 0.10$ \\
\hline Liver & $9.23 \pm 1.43$ & $9.64 \pm 0.71$ & $9.50 \pm 0.31$ & $10.03 \pm 0.53$ \\
\hline Spleen & $0.82 \pm 0.13$ & $0.84 \pm 0.12$ & $0.76 \pm 0.05$ & $0.79 \pm 0.09$ \\
\hline Kidney & $1.13 \pm 0.09$ & $1.17 \pm 0.12$ & $1.15 \pm 0.03$ & $1.18 \pm 0.10$ \\
\hline Adrenal gland & $0.022 \pm 0.002$ & $0.022 \pm 0.004$ & $0.022 \pm 0.003$ & $0.023 \pm 0.002$ \\
\hline Testis & $1.83 \pm 0.19$ & $1.93 \pm 0.14$ & $1.96 \pm 0.11$ & $2.01 \pm 0.17$ \\
\hline \multicolumn{5}{|l|}{ Female } \\
\hline Brain & $1.68 \pm 0.05$ & $1.75 \pm 0.11$ & $1.71 \pm 0.12$ & $1.71 \pm 0.08$ \\
\hline Heart & $0.72 \pm 0.06$ & $0.72 \pm 0.06$ & $0.72 \pm 0.04$ & $0.81 \pm 0.03$ \\
\hline Lung & $1.18 \pm 0.05$ & $1.15 \pm 0.05$ & $1.14 \pm 0.07$ & $1.16 \pm 0.10$ \\
\hline Liver & $5.83 \pm 0.24$ & $5.97 \pm 0.60$ & $5.72 \pm 0.38$ & $5.84 \pm 0.44$ \\
\hline Spleen & $0.58 \pm 0.06$ & $0.60 \pm 0.05$ & $0.57 \pm 0.04$ & $0.60 \pm 0.05$ \\
\hline Kidney & $0.71 \pm 0.02$ & $0.72 \pm 0.05$ & $0.71 \pm 0.03$ & $0.72 \pm 0.05$ \\
\hline Adrenal gland & $0.031 \pm 0.004$ & $0.028 \pm 0.003$ & $0.031 \pm 0.004$ & $0.029 \pm 0.004$ \\
\hline Uterus & $0.52 \pm 0.19$ & $0.43 \pm 0.07$ & $0.43 \pm 0.09$ & $0.47 \pm 0.20$ \\
\hline
\end{tabular}

Data were expressed as mean \pm SD.

There were no significantly differences among the groups. 
package was used. A $p<0.05$ indicated a statistical significance. In the bacterial reverse mutation test, the mean value and standard deviation of each group were measured and compared with the negative control groups.

\section{RESULTS}

Subacute oral toxicity study. No mortality and notable clinical signs were observed during the 28 days expo-

Table 5. Relative organ weight for rats in the 28 days oral administration of KGC-03-PS

\begin{tabular}{|c|c|c|c|c|}
\hline \multirow{2}{*}{$\begin{array}{l}\text { Relative weight } \\
(\%)\end{array}$} & \multicolumn{4}{|c|}{ Dose $(\mathrm{mg} / \mathrm{Kg})$} \\
\hline & $0(n=6)$ & $500(n=6)$ & $1000(n=6)$ & $2000(n=6)$ \\
\hline \multicolumn{5}{|l|}{ Male } \\
\hline Brain & $0.579 \pm 0.038$ & $0.566 \pm 0.028$ & $0.581 \pm 0.014$ & $0.579 \pm 0.013$ \\
\hline Heart & $0.343 \pm 0.020$ & $0.345 \pm 0.020$ & $0.367 \pm 0.026$ & $0.353 \pm 0.010$ \\
\hline Lung & $0.448 \pm 0.023$ & $0.456 \pm 0.032$ & $0.455 \pm 0.023$ & $0.439 \pm 0.030$ \\
\hline Liver & $2.795 \pm 0.346$ & $2.923 \pm 0.091$ & $2.933 \pm 0.159$ & $3.048 \pm 0.062$ \\
\hline Spleen & $0.249 \pm 0.024$ & $0.252 \pm 0.025$ & $0.235 \pm 0.012$ & $0.239 \pm 0.019$ \\
\hline Kidney & $1.129 \pm 0.095$ & $1.170 \pm 0.117$ & $1.149 \pm 0.026$ & $1.183 \pm 0.098$ \\
\hline Adrenal gland & $0.007 \pm 0.001$ & $0.007 \pm 0.001$ & $0.007 \pm 0.001$ & $0.007 \pm 0.001$ \\
\hline Testis & $0.556 \pm 0.036$ & $0.583 \pm 0.060$ & $0.607 \pm 0.029$ & $0.618 \pm 0.034$ \\
\hline \multicolumn{5}{|l|}{ Female } \\
\hline Brain & $0.839 \pm 0.031$ & $0.840 \pm 0.055$ & $0.871 \pm 0.060$ & $0.846 \pm 0.041$ \\
\hline Heart & $0.361 \pm 0.029$ & $0.360 \pm 0.015$ & $0.368 \pm 0.017$ & $0.354 \pm 0.019$ \\
\hline Lung & $0.588 \pm 0.030$ & $0.550 \pm 0.021$ & $0.581 \pm 0.042$ & $0.573 \pm 0.027$ \\
\hline Liver & $2.911 \pm 0.130$ & $2.850 \pm 0.177$ & $2.909 \pm 0.190$ & $2.891 \pm 0.167$ \\
\hline Spleen & $0.291 \pm 0.026$ & $0.290 \pm 0.029$ & $0.289 \pm 0.020$ & $0.296 \pm 0.024$ \\
\hline Kidney & $0.357 \pm 0.010$ & $0.343 \pm 0.010$ & $0.361 \pm 0.010$ & $0.355 \pm 0.013$ \\
\hline Adrenal gland & $0.015 \pm 0.002$ & $0.013 \pm 0.002$ & $0.016 \pm 0.002$ & $0.015 \pm 0.002$ \\
\hline Uterus & $0.258 \pm 0.088$ & $0.205 \pm 0.028$ & $0.219 \pm 0.047$ & $0.231 \pm 0.094$ \\
\hline
\end{tabular}

Data were expressed as mean \pm SD.

There were no significantly differences among the groups.

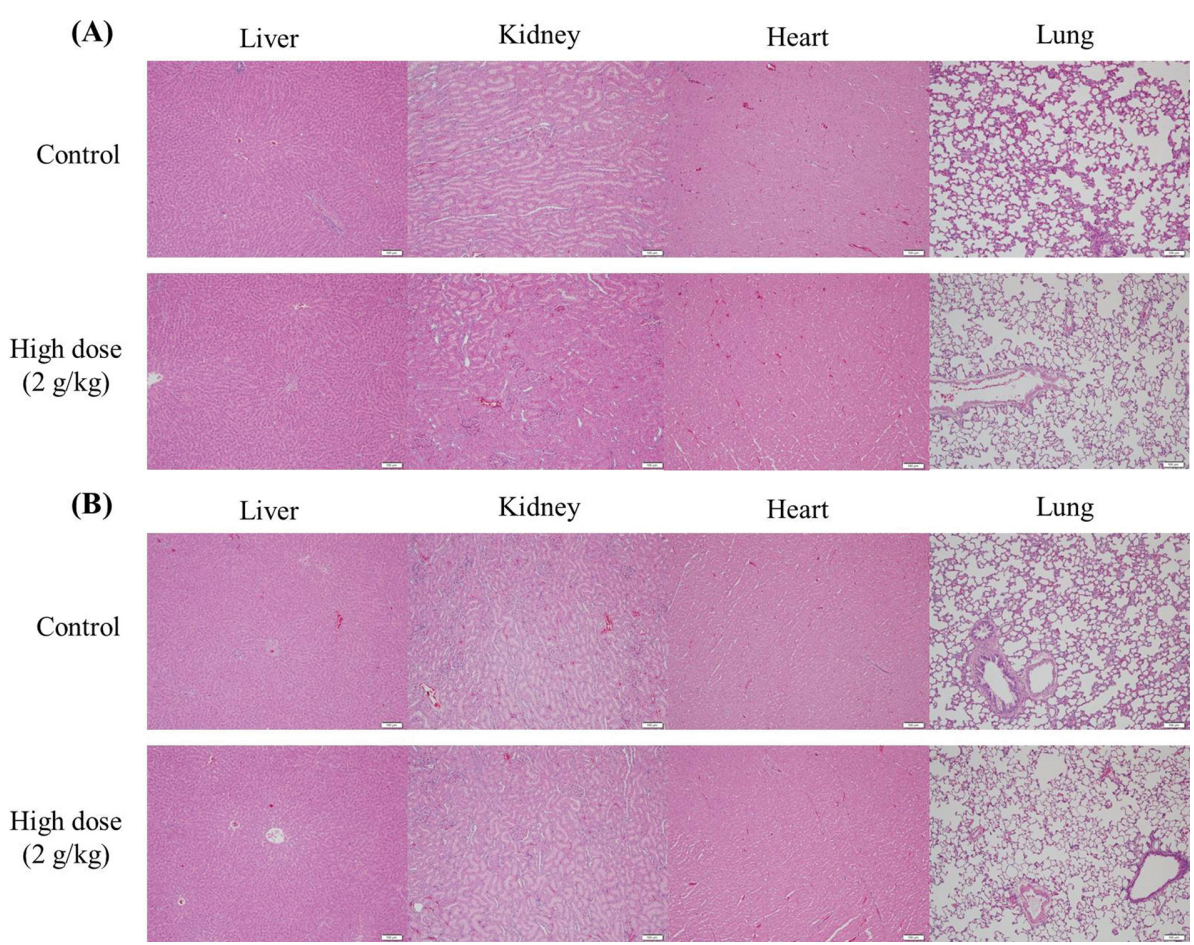

Fig. 3. Histopathological examination of male (A) and female (B) rats exposed with KGC-03-PS. There were no significant toxicological lesions both control and high dose groups. Hematoxylin \& Eosin; Magnification: 100x. 
sure period and there were no statistically significant changes in body weight and food consumption compared with control group (Fig. 1, 2).

In blood biochemistry analyses, there were statistically significant increase in glucose $(p<0.05)$ in female highdose group $(2,000 \mathrm{mg} / \mathrm{kg}$ bw) (Table 2). In hematology analyses, red blood cell significantly increased $(p<0.05)$ in male middle-dose groups $(1,000 \mathrm{mg} / \mathrm{kg} \mathrm{bw})$ compared to control group (Table 3). In addition, red blood cell count, hemoglobin, hematocrit, and platelet count significantly increased $(p<0.05)$ in female high-dose group compared to control group.

No significant organ weight changes were observed in either the absolute and relative organ weights between the experimental and control animals both sexes (Table 4, 5).

There were no any toxicological changes related with test substances in gross finding and histopathological evaluation of each organ (Fig. 3). Several non-toxicological

Table 6. Summary of histopathological findings

\begin{tabular}{|c|c|c|c|c|c|c|}
\hline \multirow{4}{*}{$\begin{array}{c}\text { Organ/ } \\
\text { Findings }\end{array}$} & \multirow{2}{*}{$\begin{array}{c}\text { Sex } \\
\text { Group }\end{array}$} & & \multicolumn{2}{|c|}{ Male } & \multicolumn{2}{|c|}{ Female } \\
\hline & & & G1 & G4 & G1 & G4 \\
\hline & Dose $(\mathrm{mg} / \mathrm{kg})$ & & 0 & 2000 & 0 & 2000 \\
\hline & No. of animals & & 6 & 6 & 6 & 6 \\
\hline \multirow[t]{2}{*}{ Kidney } & - Hyaline cast, focal, cortex & \pm & 1 & 1 & 1 & 2 \\
\hline & & No. of examined & 6 & 6 & 6 & 6 \\
\hline \multirow[t]{3}{*}{ Liver } & - Fatty degeneration, hepatocyte, focal & & 0 & 0 & 1 & 1 \\
\hline & - Inflammatory cell infiltration, focal & & 0 & 1 & 1 & 0 \\
\hline & & No. of examined & 6 & 6 & 6 & 6 \\
\hline
\end{tabular}

Table 7. Result of bacterial reverse mutation assay without $S 9$ activation

\begin{tabular}{|c|c|c|c|c|c|}
\hline \multirow{3}{*}{$\begin{array}{c}\text { Dose } \\
(\mu \mathrm{g} / \text { plate })\end{array}$} & \multicolumn{5}{|c|}{ Number of reverse mutants/plate (Mean \pm SD) } \\
\hline & \multicolumn{3}{|c|}{ Base substitution } & \multicolumn{2}{|c|}{ Frame shift } \\
\hline & TA100 & TA1535 & E. coli & TA98 & TA1537 \\
\hline 0 & $130 \pm 12$ & $10 \pm 1$ & $21 \pm 3$ & $20 \pm 2$ & $13 \pm 1$ \\
\hline 312.5 & $126 \pm 2$ & $9 \pm 1$ & $22 \pm 2$ & $22 \pm 0$ & $9 \pm 2$ \\
\hline 625 & $119 \pm 10$ & $14 \pm 4$ & $22 \pm 4$ & $22 \pm 3$ & $7 \pm 0$ \\
\hline 1250 & $142 \pm 10$ & $10 \pm 0$ & $21 \pm 4$ & $19 \pm 3$ & $9 \pm 1$ \\
\hline 2500 & $141 \pm 10$ & $10 \pm 2$ & $23 \pm 4$ & $18 \pm 1$ & $8 \pm 1$ \\
\hline 5000 & $145 \pm 4$ & $10 \pm 2$ & $19 \pm 4$ & $19 \pm 7$ & $9 \pm 1$ \\
\hline \multirow{2}{*}{$\begin{array}{l}\text { Positive control } \\
\quad(\mu \mathrm{g} / \text { plate })\end{array}$} & $\begin{array}{l}\mathrm{NaN} 3 \\
(0.5)\end{array}$ & $\begin{array}{l}\mathrm{NaN} 3 \\
(0.5)\end{array}$ & $\begin{array}{c}\text { 4-NQO } \\
(0.25)\end{array}$ & $\begin{array}{c}\text { 4-NQO } \\
(0.25)\end{array}$ & $\begin{array}{c}\text { 9-AA } \\
(50)\end{array}$ \\
\hline & $482 \pm 12$ & $220 \pm 26$ & $95 \pm 2$ & $122 \pm 3$ & $116 \pm 14$ \\
\hline
\end{tabular}

Table 8. Result of bacterial reverse mutation assay with $S 9$ activation

\begin{tabular}{|c|c|c|c|c|c|}
\hline \multirow{3}{*}{$\begin{array}{c}\text { Dose } \\
(\mu \mathrm{g} / \text { plate })\end{array}$} & \multicolumn{5}{|c|}{ Number of reverse mutants/plate (Mean \pm SD) } \\
\hline & \multicolumn{3}{|c|}{ Base substitution } & \multicolumn{2}{|c|}{ Frame shift } \\
\hline & TA100 & TA1535 & E. coli & TA98 & TA1537 \\
\hline 0 & $151 \pm 11$ & $9 \pm 2$ & $21 \pm 2$ & $27 \pm 5$ & $10 \pm 0$ \\
\hline 312.5 & $143 \pm 4$ & $12 \pm 3$ & $23 \pm 2$ & $26 \pm 3$ & $10 \pm 1$ \\
\hline 625 & $127 \pm 10$ & $11 \pm 3$ & $23 \pm 3$ & $29 \pm 3$ & $9 \pm 1$ \\
\hline 1250 & $145 \pm 6$ & $8 \pm 3$ & $21 \pm 3$ & $29 \pm 4$ & $10 \pm 2$ \\
\hline 2500 & $136 \pm 3$ & $10 \pm 3$ & $24 \pm 3$ & $30 \pm 2$ & $11 \pm 3$ \\
\hline 5000 & $126 \pm 1$ & $12 \pm 3$ & $23 \pm 6$ & $26 \pm 5$ & $10 \pm 0$ \\
\hline \multirow{2}{*}{$\begin{array}{l}\text { Positive control } \\
(\mu \mathrm{g} / \text { plate })\end{array}$} & 2-AA (2.0) & 2-AA (2.0) & $2-\mathrm{AA}(6.0)$ & $\mathrm{BaP}(1.0)$ & 2-AA (2.0) \\
\hline & $466 \pm 24$ & $83 \pm 4$ & $167 \pm 8$ & $207 \pm 12$ & $165 \pm 9$ \\
\hline
\end{tabular}


changes such as focal tubular hyaline cast in kidney cortex, and minimal macrovesicular fatty changes and inflammatory cells infiltration in liver, were observed in some control and high-dose $(2,000 \mathrm{mg} / \mathrm{kg} \mathrm{bw})$ treatment animals (Table 6).

Bacterial reverse mutation test. The results of the bacterial reverse mutation test with the five bacterial strains (TA98, 100, 1535, 1537 and E. coli WP2) for KGC-03-PS are showed in Table 7 and 8. The test was performed on KGC-03-PS at five different concentrations, from 312.5 to $5,000 \mu \mathrm{g} /$ plate, with or without S9 mix. There was no increase in the number of revertant colonies compared to its negative control with any dose or strains. The number of colonies of positive control chemicals for each tester stain was significantly higher than the negative control and within the control historical data range of our lab. Background lawn was observed and test substance precipitation and cytotoxicity was not detected all test strains.

\section{DISCUSSION}

Korean red ginseng and Salvia plebeia $\mathrm{R}$. Br. have been used as an herbal medicine worldwide for a while. The efficacy of Korean red ginseng has been proven by in vitro and in vivo studies (9-13) as well as by clinical research (33). Moreover, the various effects of Korean red ginseng such as the improvement of the immunity (34), blood circulation (35) and memory (36) as well as fatigue relief (37), anti-oxidation (38), and mitigation of menopausal symptoms in women (39) has been approved by the Korean Food and Drug administration. Salvia plebeia R. $\mathrm{Br}$. has also been shown to have potent anti-inflammatory and anti-oxidation effects described in previous studies $(15,16)$. Repeated oral toxicity and genotoxicity studies are representative safety assessments which are required as scientific safety data to register a functional food or supplementary diet ingredients. A repeated oral toxicity study is usually done following the OECD test guideline which suggests clinical, clinicopathological, and pathological examinations be done to evaluate the safety of the test article (23).

In the present study, clinical signs, body weight, and food intake were analyzed in the clinical evaluation. Particularly, body weight is used to identify the toxicological significance of a substance which is used to calculate a maximum tolerated dose (40). Korean red ginseng and Salvia plebeia $\mathrm{R}$. Br. extract mixture (KGC-03-PS) did not exhibit any toxicological changes in the clinical signs, body weight, and food intake.

Hematology and serum biochemistry were done as a clinicopathological evaluation. Hematology measures the numbers and morphology of various blood cell compo- nent and generally is used to diagnosis and monitor various diseases of the blood and bone marrow as well as of the immunologic, hemostatic, and vascular systems. Serum biochemistry is useful tool to predict the toxicological effects including liver and kidney function, and electrolyte balance. Because the results could be affected by the experimental environment and test machines, it is important to accumulate normal historical data from each institution to increase the confidence level of the test (41).

The increase in the red blood cell related index in the present study could be caused by a low oxygen level due to heart and pulmonary failure or kidney damage. However, no toxicological changes were observed in the weight of related organs, serum biochemistry and histopathological results. Moreover, the levels were within the historically normal range which suggest that KGC-03-PS has no toxicologically significant effect. In addition, decreased platelet was considered to be non-toxicological finding because it was shown in only $2,000 \mathrm{mg} / \mathrm{kg}$ treatment of female rat not in male and the count variation is mild within the historically normal range.

The statistically significant changes in glucose for the $2,000 \mathrm{mg} / \mathrm{kg}$ treatment of female rat are also considered as no toxicological effect because the changes were within the historically normal ranges and did not show a dose response relationship.

In the pathological evaluation, the necropsy organ weights and the gross and histopathological lesions were analyzed. Organ weight is considered to be an important indicator for the identification of toxicological effects of the test article in toxicology studies. In addition, the Society of Toxicologic Pathology (STP) recommends that the weights of the liver, heart, kidneys, brain, testes, and adrenal glands be measured in all general toxicology studies (42).

Information on gross and histopathological lesions provides target organ toxicity by identifying organ size and color as well as cellular changes (43). Administration of the KGC-03-PS did not induce any pathological changes in the main organs including the liver, kidney, heart, lung, and spleen.

Based on the clinical, clinicopathological, and pathological evaluations, it was shown that the 28 days repeated oral dose of the KGC-03-PS did not induce any toxic effects in the treated animals at the $2,000 \mathrm{mg} / \mathrm{kg}$ high dose under the conditions used in this study. This result suggests that the no-observed-adverse-effect level (NOAEL) of the KGC-03-PS is considered to be $2,000 \mathrm{mg} / \mathrm{kg}$ for both sexes.

The mutagenicity of KGC-03-PS was evaluated by bacterial mutation test and toxicity was not observed up to a maximum concentration of $5,000 \mu \mathrm{g} /$ plate in all tested bacterial strains (S. typhimurium TA98, TA100, TA1535, TA1537, and E.coli WP2) in presence or absence of metabolic activation. There was no difference in the number of 
revertant colonies between the KGC-03-PS treatment and negative control groups. The negative and positive control values were within historical control ranges observed in our laboratory, which assured of the test validity. These results showed that KGC-03-PS did not induce genotoxicity through bacterial reverse mutation under the conditions in the study. In conclusion, the results of this study indicate that oral doses of KGC-03-PS repeated daily for 28 days do not produce any detectable toxic effects in SD rats at dose levels up to $2,000 \mathrm{mg} / \mathrm{kg}$ bw. Thus, the NOAEL of KGC-03-PS is considered to be greater than $2,000 \mathrm{mg} / \mathrm{kg}$ bw in both sexes of SD rats. In addition, int the bacterial reverse mutation test, KGC-03-PS did not have any mutagenicity. Our results, as fundamental information for the evaluation of the subchronic or chronic toxicity of KGC-03-PS, should be useful in designing further studies on the safety and efficacy of KGC-03-PS.

\section{ACKNOWLEDGMENTS}

The authors are grateful to Korea Occupational Safety Health Research Institute (Korea) for their technical assistance to the safety tests.

\section{CONFLICT OF INTEREST}

All authors declare no conflicts of interest.

Received June 12, 2018; Revised October 2, 2018; Accepted November 30, 2018

\section{REFERENCES}

1. Jordan, S.A., Cunningham, D.G. and Marles, R. (2010) Assessment of herbal medicinal products: challenges, and opportunities to increase the knowledge base for safety assessment. Toxicol. Appl. Pharmacol., 243, 198-216.

2. Zhou, S., Chan, E., Li, S.C., Huang, M., Chen, X., Li, X., Zhang, Q. and Paxton, J.W. (2004) Predicting pharmacokinetic herb-drug interactions. Drug Metabol. Drug Interact., 20, 143-158.

3. Choi, R.J., Wong, A.S.T., Jia, W., Chang, I.M., Wong, R.N.S., Fan, T.P. and Kim, Y.S. (2015) Ginseng: a panacea linking East Asia and North America? Science, 350, S54-S56.

4. Ansari, N.A., Moinuddin, Alam, K. and Ali, A. (2009) Preferential recognition of Amadori-rich lysine residues by serum antibodies in diabetes mellitus: role of protein glycation in the disease process. Hum. Immunol., 70, 417-424.

5. Ha, K.S., Jo, S.H., Kang, B.H., Apostolidis, E., Lee, M.S., Jang, H.D. and Kwon, Y.I. (2011) In vitro and in vivo antihyperglycemic effect of 2 amadori rearrangement compounds, arginyl-fructose and arginyl-fructosyl-glucose. $J$. Food Sci., 76, H188-H193.

6. Shin, M.J., Kim, Y.S., Kwak, Y.S., Song, Y.B., Kim, Y.S. and Park, J.D. (2004) Enhancement of antitumor effects of paclitaxel (taxol) in combination with red ginseng acidic polysaccharide (RGAP). Planta Med., 70, 1033-1038.

7. Kwak, Y.S., Kyung, J.S., Kim, J.S., Cho, J.Y. and Rhee, M.H. (2010) Anti-hyperlipidemic effects of red ginseng acidic polysaccharide from Korean red ginseng. Biol. Pharm. Bull., 33, 468-472.

8. Lee, S.D., Park, S.K., Lee, E.S., Kim, H.M., Lee, C.W., Lee, K., Lee, K.H., Kang, M.R., Lee, K.S., Lee, J., Hwang, W.I. and Kim, D.C. (2010) A lipid-soluble red ginseng extract inhibits the growth of human lung tumor xenografts in nude mice. J. Med. Food, 13, 1-5.

9. Heo, J.H., Lee, S.T., Chu, K., Oh, M.J., Park, H.J., Shim, J.Y. and Kim, M. (2008) An open-label trial of Korean red ginseng as an adjuvant treatment for cognitive impairment in patients with Alzheimer's disease. Eur. J. Neurol., 15, 865868.

10. Stavro, P.M., Woo, M. and Vuksan, V. (2004) Korean red ginseng lowers blood pressure in individuals with hypertension. Am. J. Hypertens., 17, S33.

11. Byeon, S.E., Lee, J., Kim, J.H., Yang, W.S., Kwak, Y.S., Kim, S.Y., Choung, E.S., Rhee, M.H. and Cho, J.Y. (2012) Molecular mechanism of macrophage activation by red ginseng acidic polysaccharide from Korean red ginseng. Mediat. Inflamm., 2012, 732-860.

12. Yun, T.K., Zheng, S., Choi, S.Y., Cai, S.R., Lee, Y.S., Liu, X.Y., Cho, K.J. and Park, K.Y. (2010) Non-organ-specific preventive effect of long-term administration of korean red ginseng extract on incidence of human cancers. J. Med. Food, 13, 489-494.

13. Ki, S.H., Yang, J.H., Ku, S.K., Kim, S.C., Kim, Y.W. and Cho, I.J. (2013) Red ginseng extract protects against carbon tetrachloride-induced liver fibrosis. J. Ginseng Res., 37, 4553.

14. Jin, X.F., Lu, Y.H., Wei, D.Z. and Wang, Z.T. (2008) Chemical fingerprint and quantitative analysis of Salvia plebeia R.Br. by high-performance liquid chromatography. J. Pharm. Biomed. Anal., 48, 100-104.

15. Choi, J.K., Oh, H.M., Lee, S., Kwon, T.K., Shin, T.Y., Rho, M.C. and Kim, S.H. (2014) Salvia plebeian suppresses atopic dermatitis-like skin lesions. Am. J. Chinese Med., 42, 967-985.

16. Gu, L. and Weng, X. (2001) Antioxidant activity and components of Salvia plebeia R.Br.: a Chinese herb. Food Chem., 73, 299-305.

17. Ai-li, J. and Chang-hai, W. (2006) Antioxidant properties of natural components from Salvia plebeia on oxidative stability of ascidian oil. Proc. Biochem., 41, 1111-1116.

18. Choi, S.I., Cho, I.H., Han, S.H., Jeon, Y.J., Choi, J.G., Kim, J.S. and Lee, J.H. (2016) Antiobesity effects of Salvia plebeia $\mathrm{R}$. Br. extract in high-fat diet-induced obese mice. $J$. Med. Food, 19, 1048-1056.

19. Song, H., Ji, K.Y., Kim, B.K., Yang, W.K., Han, C.K., Shin, C.K., Shin, H.J., Park, Y.C., Hwang, J.S., Kang, H.S. and Kim, S.H. (2017) Respiratory protective effect of Salvia plebeian $\mathrm{R}$. Br. extracts against ambient particulate matterinduced airway inflammation. Korean J. Med. Crop Sci., 25, 269-281.

20. So, S.H., Lee, S.K., Hwang, E.I., Koo, B.S., Han, G.H., Lee, M.J., Chung, J.H. and Kim, N.M. (2008) Mechanism of Korean red ginseng and herb extract (KTNG0345) for anti- 
wrinkle activity. J. Ginseng Res., 32, 39-47.

21. Park, S.J., Lim, K.H., Noh, J.H., Jeong, E.J., Kim, Y.S., Han, B.C., Lee, S.H. and Moon, K.S. (2013) Subacute oral toxicity study of Korean red ginseng extract in Sprague-Dawley rats. Toxicol. Res., 29, 285-292.

22. Park, S.J., Noh, J.H., Jeong, E.J., Kim, Y.S., Han, B.C., Lee, S.H. and Moon, K.S. (2018) Subchronic oral toxicity study of Korean red ginseng extract in Sprague-Dawley Rats with a 4-week recovery period. Regul. Toxicol. Pharmacol., 92, 83-93.

23. OECD (2008) Guidelines for Testing of Chemical: Repeated Dose 28-Day Oral Toxicity Study in Rodents (TG 407).

24. OECD (2001) Guidelines for Testing of Chemical: Acute Oral Toxicity - Acute Toxic Class Method (TG 423).

25. Ruehl Fehlert, C., Kittel, B., Morawietz, G., Deslex, P., Keenan, C., Mahrt, C.R., Nolte, T., Robinson, M., Stuart, B.P. and Deschl, U. (2003) Revised guides for organ sampling and trimming in rats and mice--part 1. Exp. Toxic. Pathol., 55, 91-106.

26. OECD (1997) Guidelines for Testing of Chemical: Bacterial Reverse Mutation Test (TG 471).

27. Mortelmans, K. and Zeiger, E. (2000) The Ames Salmonella/ microsome mutagenicity assay. Mutat. Res., 455, 29-60.

28. Ames, B.N., McCann, J. and Yamasaki, E. (1975) Methods for detecting carcinogens and mutagens with the Salmonella/ mammalian-microsome mutagenicity test. Mutat. Res., 31, 347-364.

29. Maron, D.M. and Ames, B.N. (1983) Revised methods for the Salmonella mutagenicity test. Mutat. Res., 113, 173-215.

30. Mortelmans, K. and Riccio, E.S. (2000) The bacterial tryptophan reverse mutation assay with Escherichia coli WP2. Mutat. Res., 455, 61-69.

31. Jeong, M.H., Yang, K., Lee, C.G., Jeong, D.H., Park, Y.S., Choi, Y.J., Kim, J.S., Oh, S.J., Jeong, S.K. and Jo, W.S. (2014) In vitro genotoxicity assessment of a novel resveratrol analogue, HS-1793. Toxicol. Res., 30, 211-220.

32. Reed, D.J. (2005) Current protocols in Toxicology, Genetic Toxicology: Mutagenesis and Adduct Formation. Wiley.

33. Yun, T.K., Zheng, S., Choi, S.Y., Cai, S.R., Lee, Y.S., Liu, X.Y., Cho, K.J. and Park, K.Y. (2010) Non-organ-specific preventive effect of long-term administration of Korean red ginseng extract on incidence of human cancers. J. Med. Food, 13, 489-494.

34. Kaneko, H. and Nakanishi, K. (2004) Proof of the mysterious efficacy of ginseng: basic effects of medical ginseng, Korean red ginseng: its anti-stress action for prevention of disease. J. Pharmacol. Sci., 95, 158-162.
35. Shin, K.S., Lee, J.J., Kim, Y.I., Yu, J.Y., Park, E.S., Im, J.H., You, S.H., Oh, K.W., Lee, M.K., Wee, J.J., Kim, Y.S. and Yun, Y.P. (2007) Effect of Korean red ginseng extract on blood circulation in healthy volunteers: a randomized, double-blind, placebo-controlled trial. J. Ginseng. Res., 31, 109116.

36. Kennedy, D.O., Reay, J.L. and Scholey, A.B. (2007) Effect of 8 weeks administration of Korean red ginseng extract on the mood and cognitive performance of healthy individuals. J. Ginseng Res., 31, 34-43.

37. Yoon, S.J., Kim, K.H., Kim, C.J., Park, H.C., Kang, K.H., Kim, M.J., Kang, S.M., Kwak, U.H. and Kim, H.J. (2008) Effects of red ginseng supplementation on aerobic.anaerobic performance, central and peripheral fatigue. J. Ginseng Res., 32, 210-219.

38. Kim, J.Y., Park, J.Y., Kang, H.J., Kim, O.Y. and Lee, J.H. (2012) Beneficial effects of Korean red ginseng on lymphocyte DNA damage, antioxidant enzyme activity, and LDL oxidation in healthy participants: a randomized, doubleblind, placebo-controlled trial. Nutr. J., 11, 47.

39. Kim, S.Y., Seo, S.K., Choi, Y.M., Jeon, Y.E., Lim, K.J., Cho, S., Choi, Y.S. and Lee, B.S. (2012) Effects of red ginseng supplementation on menopausal symptoms and cardiovascular risk factors in postmenopausal women: a double-blind randomized controlled trial. Menopause, 19, 461-466.

40. Chapman, K., Sewell, F., Allais, L., Delongeas, J.L., Donald, E., Festag, M., Kervyn, S., Ockert, D., Nogues, V., Palmer, H., Popovic, M., Roosen, W., Schoenmakers, A., Somers, K., Stark, C., Stei, P. and Robinson, S. (2013) A global pharmaceutical company initiative: An evidence-based approach to define the upper limit of body weight loss in short term toxicity studies. Regul. Toxicol. Pharmacol., 67, 27-38.

41. Han, Z.Z., Xu, H.D., Kim, K.H., Ahn, T.H., Bae, J.S., Lee, J.Y., Gil, K.H., Lee, J.Y., Woo, S.J., Yoo, H.J., Lee, H.K., Kim, K.H., Park, C.K., Zhang, H.S. and Song, S.W. (2010) Reference data of the main physiological parameters in control Sprague-Dawley rats from pre-clinical toxicity studies. Lab. Anim. Res., 26, 153-164.

42. Sellers, R.S., Morton, D., Michael, B., Roome, N., Johnson, J.K., Yano, B.L., Perry, R. and Schafer, K. (2007) Society of toxicologic pathology position paper: Organ weight recommendations for toxicology studies. Toxicol. Pathol., 35, 751755.

43. Crissman, J.W., Goodman, D.G., Hildebrandt, P.K., Maronpot, R.R., Prater, D.A., Riley, J.H., Seaman, W.J. and Thake, D.C. (2004) Best practices guideline: toxicologic histopathology. Toxicol. Pathol., 32, 126-131. 